\title{
A Review of Devices For Integrated Silicon Photonic SWITCHES
}

\section{A PREPRINT}

\author{
Akhilesh S. P. Khope \\ Microsoft Corporation \\ One Microsoft Way \\ Redmond, WA 98052 \\ akhkhope@gmail.com
}

June 6, 2021

\begin{abstract}
In this paper, we review devices used in silicon photonic switches. Devices in switches are divided into active and passive devices. Active devices consist of microring resonator, contra directional couplers, mach zhender switches. Passive devices consist of waveguide crossings and arrayed waveguide gratings. We also list the state of the art in devices in a comparison table.
\end{abstract}

Keywords Switching $\cdot$ Photonic Integrated Circuits $\cdot$ Optical Switching Devices

\section{Introduction}

Optical switching in datacenters enable reconfigurable datacenter networks [1]. Space switches are reported in [2, 3, 4, 5, 6, 7, 8, 9, 10, 11, 12, 13, 14, 15, 16, 17]. Some notable demonstrations of wavelength selective switches (WSS) are reported in [18, 19, 20, 21, 22, 23, 24]. We proposed a WSS in [25] and later demonstrate in [26, 27, 18, 28, 29]. WSS with half the number of devices and with multicasting is also reported in [30,31]. For details on WSS, refer to the review in [32]. In this paper, we give a short overview of different optical devices used in integrated photonic switches. The major contributions of this paper are as follows:

1. If one were to use the devices mentioned in this paper in the switches, the optical loss is lower than one achieved with typical devices found in literature.

2. This review will help researchers to focus on system level design than starting from deigning devices while building bigger systems.

3. The device designs reported in this paper are freely available and can be easily reproduced as compared with black box foundry process design kit (PDK).

A silicon photonics PDK is reported in [33]. A CMOS compatible PDK is reported in [34]. Devices from both these efforts and other papers are combined in this review. In Section 2, we give an overview of tuning mechanism used in devices. In Section 3, we review state of the art in Active devices. In Section 4, we review state of the art in Passive devices. In Section 5 Discussions, we summarize the findings in a table.

\section{Tuning Mechanism}

In this section, we consider tuning mechanism used in integrated optical filters and switches. We consider three types of tuning mechanisms. Thermal tuning, electro-optic tuning and tuning based on kerr effect. Thermal tuning tunes on the order of 10s of $\mathrm{nm}$ and is useful for filters when tuning across multiple channels is required [35, 36, 37]. In thermal tunning, current is injected into a resistor near which can be a doped silicon waveguide, Tungsten wiring or Ni-silicide. 
Tuning time is on the order of $\mu s$. Thermal undercut of the ring resonator is reported in [38]. This arrangement has a lower power consumption than other MRRs. A comparison of silicon photonic heaters is provided in [39]. Adiabatic designs reduce overlap between optical mode and the heater and reduce optical loss [40].

The heater vias are the bottleneck in these designs and burnout when we pass a lot of current. We show that tuning times for MRR are on the order of 10s of $\mu s$ as compared to $1 \mu s$ as tuning across many wavelengths is required[18]. This tuning time is much smaller as compared with time required for arbitration in a software defined network (SDN) (1s).

Optical loss is much higher for electo-optic tuning. There are multiple ways to tune MRR with electro optic tuning. We consider only carrier-injection tuning [41] as it is relevant for switches. In carrier-injection tuning we pass current which changes the refractive index of the waveguide. This leads to a change in resonant wavelength of the MRR.

We review active and passive devices used in switches in the next two sections.

\section{Active Devices}

We review active devices used in switches in this section. Active devices have thermal heaters or carrier injection phase shifters.

\subsection{MRR}

Microring resonator (MRR) is a device with a circular waveguide coupled to a straight waveguide or waveguides used for switching or filtering a particular wavelength from a WDM channel. MRRs can be either first order, higher order coupled ring resonators [42, 43] or series coupled [44]. A flat top filter shape with high out of band rejection is required for lower crosstalk in a switch. Higher order rings have these features and are sensitive to fabrication variation: optical waveguide shape, coupling gap and thickness uniformity of the wafer. A review of MRRs is reported in [45]. Theoretical approach for coupled resonator optical waveguides (CROW) is reported in [42].

(a)

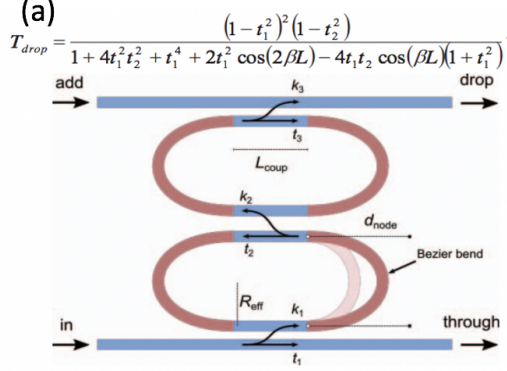

(b)

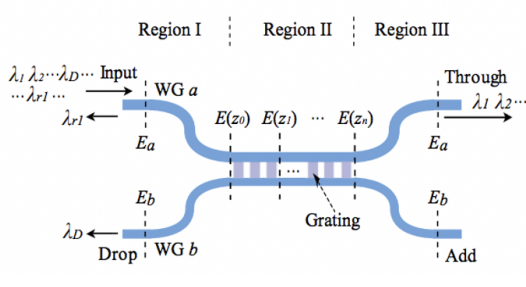

(c)

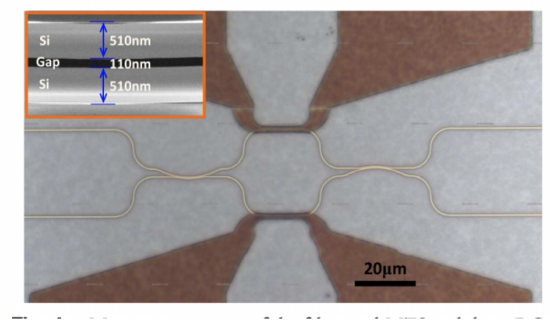

Figure 1: (a) second order MRR with thermal tuning and low innsertion loss [46], (b) contra directional coupler [47], (c) MZI[48]

Ring resonators in silicon photonics process have a footprint of $10 \mu m \times 10 \mu m$ for FSR greater than $20 \mathrm{~nm}$. Switching speed of $1 \mu s$ are reported by thermal tuning [40] and $1 n s$ by electro optic tuning [49]. Thermal tuning tunes farther than electro-optic tuning with minimal optical loss. We reported a $15 \mu \mathrm{s}$ rise time to tune across the multiple WDM channels of the MRR in [18]. In most papers, 1/e switching time is reported but for wavelength selective switching, tuning across whole FSR is required which can be more than $30 \mathrm{~nm}$ for 8 channel at $400 \mathrm{GHz}$ spacing. MRRs in InP and $\mathrm{SiN}$ platform have higher footprint and power consumption due to their size and lower index contrast.

\subsection{Contra directional couplers}

Contra directional couplers (CDC) are grating filters that can be designed for a very wide filter shape. The filtered wavelengths propagate in opposite direction to the input direction and rest of the wavelengths pass to the through port. These filters can be used as a broadband filter to drop multiple WDM channel and have infinite free spectral range. Switches with very high port counts can be designed with these devices. Apodized contra directional couplers with thermal tuning are reported in [47, 50]. In apodization, the strength of the grating follows a gaussian curve. Apodization can produce flat top filter shapes with higher out of band rejection. A tunable MRR-contra directional switch is reported in [51]. Contra directional couplers with heaters are also reported in [52]. 
A PREPRINT - JUNE 6, 2021

\subsection{MZI}

Mach zhender interferometer (MZI) is a device with two directional couplers and a waveguide section between them. These switches have a higher footprint than (MRR) and can be tuned with carrier -injection phase shifters or thermal tuning. A low power MZI with thermal tuning is reported in [48]. A fast nanosecond MZ switch is reported in [53]. Low power thermal tuning with adiabatic bends and integrated thermal tuners is reported in [54]. Many switching networks on chip use broadband directional couplers or multi mode interferometers (MMI). These switches are used to build bigger integrated fiber switches that switch all wavelength division multiplexing (WDM) channels from a fiber.

\section{Passive elements}

In this section, we provide an overview of passive elements.

\subsection{AWGR}

Arrayed waveguide grating router (AWGR) consists of input and output waveguides, two free-propagation slab regions, and arrayed waveguides where each neighboring waveguide has a constant path length difference. Each input port of the AWGR can communicate with different output port with specific WDM wavelengths based on the wavelength routing function. Since AWGR is an interferometric device, the phase errors cause a degraded crosstalk. Silicon nitride (SiN) AWGR is superior to silicon AWGR in mitigating the degraded crosstalk since the variation of the average effective index of the arrayed waveguides is lower. The state-of-the-art SiN AWGR can have the adjacent and non-adjacent channel crosstalk as low as $-39 \mathrm{~dB}$ and $-33.5 \mathrm{~dB}$, respectively [55].

(a)

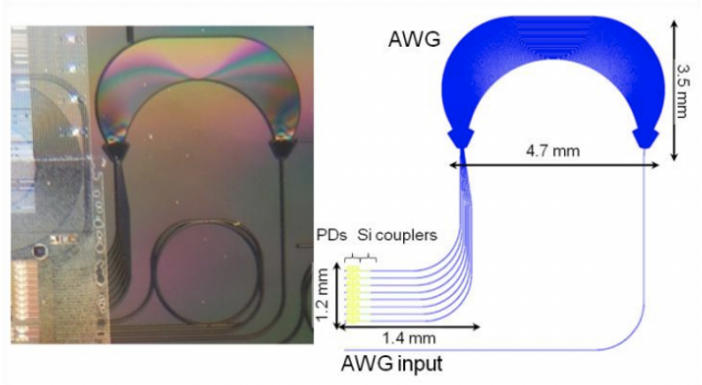

(b)

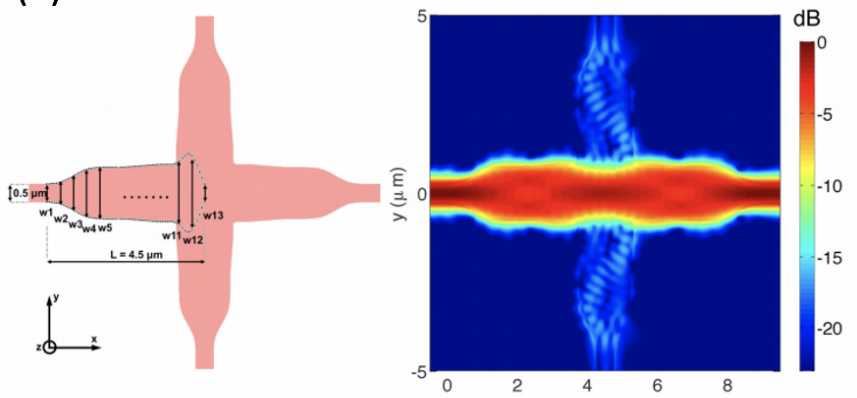

Figure 2: (a) ultralow crosstalk AWG [55], (b) Waveguide Crossing [56]

\subsection{Waveguide crossing}

Waveguide crossing is a device used for crossing optical waveguides [57]. Optical waveguide width is higher at the junction to focus the mode at the intersection of the two waveguides to minimize optical scatering. Waveguide crossings with loss of less than $0.02 \mathrm{~dB}$ and crosstalk less than $-37 \mathrm{~dB}$ are reported for SOI platform in [56]. This device is $9 \mu m \times 9 \mu m$ These waveguide crossings use optimization algorithms to change the layout of the crossing. Straight waveguide crossing incur a larger optical loss. Other notable crossings are reported in [58]. Silicon and silicon nitride trilayer waveguide crossings are reported in [59]. These gratings have greater size as compared with SOI waveguide crossings but enable more complex photonic integrated circuits.

\section{Discussion}

The following table summarizes the best devices and we bold their best metrics. 
A PREPRINT - JUNE 6, 2021

\begin{tabular}{|c|c|c|c|c|c|}
\hline Device & Size & $\begin{array}{c}\text { Power } \\
\text { Consumption }\end{array}$ & $\begin{array}{l}\text { Switching } \\
\text { Speed }\end{array}$ & $\begin{array}{c}\text { Tuning } \\
\text { Mechanism }\end{array}$ & Comments \\
\hline MRR [36] & $8 \mu m \times 10 \mu m$ & $2.4 \mathrm{~mW}$ & $170 \mu \mathrm{s}$ & Thermal & - \\
\hline MRR [40] & - & $20 \mathrm{~mW}$ & $1 \mu s$ & Thermal & - \\
\hline Single $\lambda$ MZI [54] & $200 \mu m$ length & 12.7mW $\pi$ phase & $2.4 \mu s$ & Thermal & - \\
\hline Broadband MZI [48] & $100 \mu m$ length & - & - & Thermal & $\begin{array}{l}\text { 1dB IL } \\
140 \mathrm{~nm}\end{array}$ \\
\hline CDC [52] & $312 \mu m$ & - & - & Thermal & $\begin{array}{c}55 \mathrm{~dB} \text { contrast } \\
<0.5 \mathrm{~dB} \text { loss }\end{array}$ \\
\hline AWGR [55] & - & - & - & - & -33.5 dB Crosstalk \\
\hline Waveguide Crossing [56] & $9 \mu m \times 9 \mu m$ & - & - & - & $0.02 \mathrm{~dB}$ \\
\hline
\end{tabular}

\section{Conclusion}

We report state of the art devices in integrated silicon photonic switches. The devices from the table can be easily reproduced and fabricated in a silicon photonic foundry.

Acknowledgements: Author thanks Xian Xiao for insightful discussions on AWGRs.

\section{References}

[1] Adel AM Saleh, Akhilesh SP Khope, John E Bowers, and Rod C Alferness. Elastic wdm switching for scalable data center and hpc interconnect networks. In 2016 21st OptoElectronics and Communications Conference $($ OECC) held jointly with 2016 International Conference on Photonics in Switching (PS), pages 1-3. IEEE, 2016.

[2] Liang Yuan Dai, Yu-Han Hung, Qixiang Cheng, and Keren Bergman. Experimental demonstration of pam-4 transmission through microring silicon photonic clos switch fabric. pages M1H-3, 2020.

[3] Ryotaro Konoike, Keijiro Suzuki, Shu Namiki, Hitoshi Kawashima, and Kazuhiro Ikeda. Ultra-compact silicon photonics switch with high-density thermo-optic heaters. Optics express, 27(7):10332-10342, 2019.

[4] R Stabile, A Albores-Mejia, and KA Williams. Monolithic active-passive 16× 16 optoelectronic switch. Optics letters, 37(22):4666-4668, 2012.

[5] Lei Qiao, Weijie Tang, and Tao Chu. $32 \times 32$ silicon electro-optic switch with built-in monitors and balanced-status units. Scientific Reports, 7:42306, 2017.

[6] Lei Qiao, Weijie Tang, and Tao Chu. 16× 16 non-blocking silicon electro-optic switch based on mach-zehnder interferometers. In Optical Fiber Communication Conference, pages Th1C-2. Optical Society of America, 2016.

[7] Dritan Celo, Dominic J Goodwill, Jia Jiang, Patrick Dumais, Chunshu Zhang, Fei Zhao, Xin Tu, Chunhui Zhang, Shengyong Yan, Jifang He, et al. 32× 32 silicon photonic switch. In 201621 st OptoElectronics and Communications Conference (OECC) held jointly with 2016 International Conference on Photonics in Switching $(P S)$, pages 1-3. IEEE, 2016.

[8] Liangjun Lu, Shuoyi Zhao, Linjie Zhou, Dong Li, Zuxiang Li, Minjuan Wang, Xinwan Li, and Jianping Chen. $16 \times 16$ non-blocking silicon optical switch based on electro-optic mach-zehnder interferometers. Optics express, 24(9):9295-9307, 2016.

[9] Zeqin Lu, Dritan Celo, Hamid Mehrvar, Eric Bernier, and Lukas Chrostowski. High-performance silicon photonic tri-state switch based on balanced nested mach-zehnder interferometer. Scientific reports, 7(1):1-7, 2017.

[10] Nicolas Dupuis, Benjamin G Lee, Alexander V Rylyakov, Daniel M Kuchta, Christian W Baks, Jason S Orcutt, Douglas M Gill, William MJ Green, and Clint L Schow. Design and fabrication of low-insertion-loss and low-crosstalk broadband $2 \times 2$ mach-zehnder silicon photonic switches. Journal of Lightwave Technology, 33(17):3597-3606, 2015.

[11] Benjamin G Lee, Aleksandr Biberman, Po Dong, Michal Lipson, and Keren Bergman. All-optical comb switch for multiwavelength message routing in silicon photonic networks. IEEE Photonics Technology Letters, 20(10):767$769,2008$.

[12] Yurii Vlasov, William MJ Green, and Fengnian Xia. High-throughput silicon nanophotonic wavelength-insensitive switch for on-chip optical networks. nature photonics, 2(4):242, 2008. 
[13] Keijiro Suzuki, Ryotaro Konoike, Junichi Hasegawa, Satoshi Suda, Hiroyuki Matsuura, Kazuhiro Ikeda, Shu Namiki, and Hitoshi Kawashima. Low insertion loss and power efficient $32 \times 32$ silicon photonics switch with extremely-high- $\delta$ plc connector. In Optical Fiber Communication Conference, pages Th4B-5. Optical Society of America, 2018.

[14] Qixiang Cheng, Adrian Wonfor, Richard V Penty, and Ian H White. Scalable, low-energy hybrid photonic space switch. Journal of Lightwave Technology, 31(18):3077-3084, 2013.

[15] Qixiang Cheng, Sébastien Rumley, Meisam Bahadori, and Keren Bergman. Photonic switching in high performance datacenters. Optics express, 26(12):16022-16043, 2018.

[16] Hao Yang, Qixiang Cheng, Rui Chen, and Keren Bergman. Polarization-diversity microring-based optical switch fabric in a switch-and-select architecture. pages Th3B-2, 2020.

[17] Yishen Huang, Qixiang Cheng, Yu-Han Hung, Hang Guan, Xiang Meng, Ari Novack, Matthew Streshinsky, Michael Hochberg, and Keren Bergman. Multi-stage $8 \times 8$ silicon photonic switch based on dual-microring switching elements. Journal of Lightwave Technology, 2019.

[18] Akhilesh SP Khope, Mitra Saeidi, Raymond Yu, Xinru Wu, Andrew M Netherton, Yuan Liu, Zeyu Zhang, Yujie Xia, Garey Fleeman, Alexander Spott, et al. Multi-wavelength selective crossbar switch. Optics express, 27(4):5203-5216, 2019.

[19] Tae Joon Seok, Jianheng Luo, Zhilei Huang, Kyungmok Kwon, Johannes Henriksson, John Jacobs, Lane Ochikubo, Richard S Muller, and Ming C Wu. Silicon photonic wavelength cross-connect with integrated mems switching. APL Photonics, 4(10):100803, 2019.

[20] X. Xiao, R. Proietti, G. Liu, H. Lu, Y. Zhang, and S. J. B. Yoo. Multi-fsr silicon photonic flex-lions module for bandwidth-reconfigurable all-to-all optical interconnects. Journal of Lightwave Technology, 38(12):3200-3208, 2020.

[21] Yishen Huang, Qixiang Cheng, Anthony Rizzo, and Keren Bergman. High-performance microring-assisted space-and-wavelength selective switch. pages Th2A-7, 2020.

[22] Xian Xiao, Roberto Proietti, Sebastian Werner, Pouya Fotouhi, and SJ Ben Yoo. Flex-lions: A scalable silicon photonic bandwidth-reconfigurable optical switch fabric. pages 1-3, 2019.

[23] R Stabile, A Rohit, and KA Williams. Monolithically integrated $8 \times 8$ space and wavelength selective cross-connect. Journal of Lightwave Technology, 32(2):201-207, 2014.

[24] Abhinav Rohit, Jeroen Bolk, Xaveer JM Leijtens, and Kevin A Williams. Monolithic nanosecond-reconfigurable 4 x 4 space and wavelength selective cross-connect. Journal of Lightwave Technology, 30(17):2913-2921, 2012.

[25] Akhilesh SP Khope, Adel AM Saleh, John E Bowers, and Rod C Alferness. Elastic wdm crossbar switch for data centers. In 2016 IEEE Optical Interconnects Conference (OI), pages 48-49. IEEE, 2016.

[26] Akhilesh SP Khope, Takako Hirokawa, Andrew M Netherton, Mitra Saeidi, Yujie Xia, Nicolas Volet, Clint Schow, Roger Helkey, Luke Theogarajan, Adel AM Saleh, et al. On-chip wavelength locking for photonic switches. Optics letters, 42(23):4934-4937, 2017.

[27] Akhilesh SP Khope, Andrew M Netherton, Takako Hirokawa, Nicolas Volet, Eric J Stanton, Clint Schow, Roger Helkey, Adel AM Saleh, John E Bowers, and Rod C Alferness. Elastic wdm optoelectronic crossbar switch with on-chip wavelength control. In Photonics in Switching, pages PTh1D-3. Optical Society of America, 2017.

[28] Akhilesh SP Khope, Songtao Liu, Zeyu Zhang, Andrew M Netherton, Rebecca L Hwang, Aaron Wissing, Jesus Perez, Franklin Tang, Clint Schow, Roger Helkey, et al. $2 \lambda$ switch. Optics Letters, 45(19):5340-5343, 2020.

[29] Akhilesh S.P. Khope, Songtao Liu, Andy Netherton, Zeyu Zhang, Sairaj Khope, Roger Helkey, Adel A.M. Saleh, Rod C. Alferness, and John E. Bowers. Experiments on multiwavelength selective crossbar switches. In 2020 International Conference on Information Science and Communications Technologies (ICISCT), pages 1-5, 2020.

[30] Akhilesh SP Khope, Roger Helkey, Songtao Liu, Sairaj Khope, Rod C Alferness, Adel AM Saleh, and John E Bowers. Scalable multicast hybrid broadband-crossbar wavelength selective switch: proposal and analysis. Optics Letters, 46(2):448-451, 2021.

[31] Akhilesh S. P. Khope, Roger Helkey, Songtao Liu, Adel A. M. Saleh, Rod C. Alferness, and John E. Bowers. A scalable multicast hybrid broadband crossbar wavelength selective switch for datacenters. In 2021 IEEE 11th Annual Computing and Communication Workshop and Conference (CCWC), pages 1585-1587, 2021.

[32] Akhilesh SP Khope, Anirban Samanta, Xian Xiao, Ben Yoo, and John E Bowers. Review of integrated photonic elastic wdm switches for data centers. arXiv preprint arXiv:2105.14934, 2021. 
A PREPRINT - JUNE 6, 2021

[33] Lukas Chrostowski, Jonas Flueckiger, Charlie Lin, Michael Hochberg, James Pond, Jackson Klein, John Ferguson, and Chris Cone. Design methodologies for silicon photonic integrated circuits. In Smart Photonic and Optoelectronic Integrated Circuits XVI, volume 8989, page 89890G. International Society for Optics and Photonics, 2014.

[34] Christophe Galland, Ari Novack, Yang Liu, Ran Ding, Michael Gould, Tom Baehr-Jones, Qi Li, Yisu Yang, Yangjin Ma, Yi Zhang, et al. A cmos-compatible silicon photonic platform for high-speed integrated optoelectronics. In Integrated Photonics: Materials, Devices, and Applications II, volume 8767, page 87670G. International Society for Optics and Photonics, 2013.

[35] Joris Van Campenhout, William MJ Green, Solomon Assefa, and Yurii A Vlasov. Integrated nisi waveguide heaters for cmos-compatible silicon thermo-optic devices. Optics Letters, 35(7):1013-1015, 2010.

[36] Po Dong, Wei Qian, Hong Liang, Roshanak Shafiiha, Dazeng Feng, Guoliang Li, John E Cunningham, Ashok V Krishnamoorthy, and Mehdi Asghari. Thermally tunable silicon racetrack resonators with ultralow tuning power. Optics express, 18(19):20298-20304, 2010.

[37] Adil Masood, M Pantouvaki, D Goossens, G Lepage, P Verheyen, Dries Van Thourhout, P Absil, and Wim Bogaerts. Cmos-compatible tungsten heaters for silicon photonic waveguides. In The 9th International Conference on Group IV Photonics (GFP), pages 234-236. IEEE, 2012.

[38] John E Cunningham, Ivan Shubin, Xuezhe Zheng, Thierry Pinguet, Attila Mekis, Ying Luo, Hiren Thacker, Guoliang Li, Jin Yao, Kannan Raj, et al. Highly-efficient thermally-tuned resonant optical filters. Optics express, 18(18):19055-19063, 2010.

[39] Adil Masood, Marianna Pantouvaki, Guy Lepage, Peter Verheyen, Joris Van Campenhout, Philippe Absil, Dries Van Thourhout, and Wim Bogaerts. Comparison of heater architectures for thermal control of silicon photonic circuits. In 10th International Conference on Group IV Photonics, pages 83-84. IEEE, 2013.

[40] Michael R Watts, William A Zortman, Douglas C Trotter, Gregory N Nielson, David L Luck, and Ralph W Young. Adiabatic resonant microrings (arms) with directly integrated thermal microphotonics. In Conference on Lasers and Electro-Optics, page CPDB10. Optical Society of America, 2009.

[41] Chin-Hui Chen, Cheng Li, Ayman Shafik, Marco Fiorentino, Patrick Chiang, Samuel Palermo, and Ray Beausoleil. A wdm silicon photonic transmitter based on carrier-injection microring modulators. In 2014 Optical Interconnects Conference, pages 121-122. IEEE, 2014.

[42] Joyce KS Poon, Jacob Scheuer, Shayan Mookherjea, George T Paloczi, Yanyi Huang, and Amnon Yariv. Matrix analysis of microring coupled-resonator optical waveguides. Optics express, 12(1):90-103, 2004.

[43] Brent E Little, Sai T Chu, John V Hryniewicz, and Philippe P Absil. Filter synthesis for periodically coupled microring resonators. Optics Letters, 25(5):344-346, 2000.

[44] Yuta Goebuchi, Tomoyuki Kato, and Yasuo Kokubun. Optimum arrangement of high-order series-coupled microring resonator for crosstalk reduction. Japanese journal of applied physics, 45(7R):5769, 2006.

[45] Paolo Pintus, Michael Hofbauer, Costanza L Manganelli, Maryse Fournier, Sarat Gundavarapu, Olivier Lemonnier, Fabrizio Gambini, Laetitia Adelmini, Carl Meinhart, Christophe Kopp, et al. Pwm-driven thermally tunable silicon microring resonators: Design, fabrication, and characterization. Laser \& Photonics Reviews, 13(9):1800275, 2019.

[46] CL Manganelli, P Pintus, F Gambini, D Fowler, M Fournier, C Kopp, F Di Pasquale, and CJ Oton. Design of coupled micro-ring resonators for silicon photonic switching matrices. In 2016 IEEE Optical Interconnects Conference (OI), pages 84-85. IEEE, 2016.

[47] Wei Shi, Xu Wang, Charlie Lin, Han Yun, Yang Liu, Tom Baehr-Jones, Michael Hochberg, Nicolas AF Jaeger, and Lukas Chrostowski. Silicon photonic grating-assisted, contra-directional couplers. Optics express, 21(3):36333650, 2013.

[48] Sitao Chen, Yaocheng Shi, Sailing He, and Daoxin Dai. Low-loss and broadband $2 \times 2$ silicon thermo-optic mach-zehnder switch with bent directional couplers. Optics letters, 41(4):836-839, 2016.

[49] Qianfan Xu, Bradley Schmidt, Sameer Pradhan, and Michal Lipson. Micrometre-scale silicon electro-optic modulator. nature, 435(7040):325-327, 2005.

[50] Wei Shi, Han Yun, Charlie Lin, Mark Greenberg, Xu Wang, Yun Wang, Sahba Talebi Fard, Jonas Flueckiger, Nicolas AF Jaeger, and Lukas Chrostowski. Ultra-compact, flat-top demultiplexer using anti-reflection contradirectional couplers for cwdm networks on silicon. Optics express, 21(6):6733-6738, 2013.

[51] Kazuhiro Ikeda, Keijiro Suzuki, Ryotaro Konoike, and Hitoshi Kawashima. Silicon photonics wavelength selective switch with unlimited free spectral range. Journal of Lightwave Technology, 38(12):3268-3272, 2020. 
[52] Jonathan St-Yves, Hadi Bahrami, Philippe Jean, Sophie LaRochelle, and Wei Shi. Widely bandwidth-tunable silicon filter with an unlimited free-spectral range. Optics letters, 40(23):5471-5474, 2015.

[53] Benjamin G Lee, Alexander V Rylyakov, William MJ Green, Solomon Assefa, Christian W Baks, Renato Rimolo-Donadio, Daniel M Kuchta, Marwan H Khater, Tymon Barwicz, Carol Reinholm, et al. Monolithic silicon integration of scaled photonic switch fabrics, cmos logic, and device driver circuits. Journal of Lightwave Technology, 32(4):743-751, 2013.

[54] Michael R Watts, Jie Sun, Christopher DeRose, Douglas C Trotter, Ralph W Young, and Gregory N Nielson. Adiabatic thermo-optic mach-zehnder switch. Optics letters, 38(5):733-735, 2013.

[55] Molly Piels, Jared F Bauters, Michael L Davenport, Martijn JR Heck, and John E Bowers. Low-loss silicon nitride awg demultiplexer heterogeneously integrated with hybrid iii-v/silicon photodetectors. Journal of lightwave technology, 32(4):817-823, 2013.

[56] Yangjin Ma, Yi Zhang, Shuyu Yang, Ari Novack, Ran Ding, Andy Eu-Jin Lim, Guo-Qiang Lo, Tom BaehrJones, and Michael Hochberg. Ultralow loss single layer submicron silicon waveguide crossing for soi optical interconnect. Optics express, 21(24):29374-29382, 2013.

[57] Wim Bogaerts, Pieter Dumon, Dries Van Thourhout, and Roel Baets. Low-loss, low-cross-talk crossings for silicon-on-insulator nanophotonic waveguides. Optics letters, 32(19):2801-2803, 2007.

[58] Sailong Wu, Xin Mu, Lirong Cheng, Simei Mao, and HY Fu. State-of-the-art and perspectives on silicon waveguide crossings: A review. Micromachines, 11(3):326, 2020.

[59] Wesley D Sacher, Jared C Mikkelsen, Patrick Dumais, Jia Jiang, Dominic Goodwill, Xianshu Luo, Ying Huang, Yisu Yang, Antoine Bois, Patrick Guo-Qiang Lo, et al. Tri-layer silicon nitride-on-silicon photonic platform for ultra-low-loss crossings and interlayer transitions. Optics express, 25(25):30862-30875, 2017. 